\title{
Contribution à l'estimation des substances appauvrissant la couche d'ozone (SAO) au Togo de 2000 à 2005
}

\author{
SABI Kokou - Ayité-Lô N. AJAVON \\ Université de Lomé Faculté des sciences \\ Laboratoire de chimie Atmosphérique \\ BP I5I5 - Lomé - TOGO
}

Pour toute correspondance : sabikokou@yahoo.fr

\begin{abstract}
Résumé
Nos estimations, réalisées dans le cadre des activités de recherche du Laboratoire de chimie atmosphérique de la Faculté des sciences de l'université de Lomé, sont une contribution à la concrétisation de la volonté du gouvernement togolais qui a ratifié la Convention de Vienne et le Protocole de Montréal relatifs aux substances appauvrissant la couche d'ozone (SAO) ainsi que tous ses amendements.

Au titre de l'année 2005, la consommation du Togo en SAO est de 934,754 tonnes PAO soit 0,000।79 tonnes PAO par habitant. Les SAO consommées proviennent exclusivement de l'importation qui est soit légale soit illicite. Utilisées essentiellement pour la réfrigération et la climatisation, les SAO se repartissent comme suit :

- 0,096 tonne métrique de CFC-II ;

- 916,704 tonnes métriques de CFC-12 ;

— 326,437 tonnes métriques de HCFC-22.
\end{abstract}

Si pour d'autres secteurs d'activité il n'existe pas de données accessibles, la porosité de nos frontières permet de soupçonner l'utilisation d'autres SAO. C'est le cas par exemple des halons et du bromure de méthyle.

Le Togo peut contrôler et revoir à la baisse sa consommation en SAO par l'amélioration du Programme de Pays qui puisse aboutir à d'autres possibilités de projets de financement.

\section{Mots clés}

SAO : substances appauvrissant la couche d'ozone

PAO : potentiel d'appauvrissement d'ozone

CFC : chlorofluorocarbure

\section{Introduction}

Les émissions de produits chimiques d'origine industrielle connus sous le terme de substances appauvrissant la couche d'ozone (SAO) réduisent la couche d'ozone stratosphérique qui protège la vie de la planète des effets nocifs de la radiation ultraviolette du soleil. L'appauvrissement de la couche d'ozone stratosphérique peut entraîner des brûlures solaires et des cancers de la peau, des cas de cataracte, la diminution de la résistance humaine et animale aux infections, la réduction de la taille des plantes donc de la production, le réchauffement général de la planète et l'accroissement des modifications climatiques. De ce fait, la communauté internationale a pris des mesures par l'adoption du Protocole de Montréal relatif aux SAO. Ce traité engage les pays à protéger la couche d'ozone par suppression graduelle de la production et de la consommation des SAO selon un calendrier précis et notre travail n'est qu'une contribution à ce protocole.

\section{Méthode}

Nos travaux sont guidés par le Manuel sur la communication réalisé par le PNUE. II propose quatre tâches et une bonne pratique, à savoir la détermination des obligations du pays, la collecte des données, la vérification de l'exactitude et de la cohérence des données, la communication et la vérification de l'état de conformité du pays.

Pour atteindre ces objectifs, nous avons mené nos enquêtes essentiellement dans la ville de Lomé.

Nous avons estimé :

- la consommation de SAO par habitant

- la consommation en tonnes de valeurs pondérées des SAO (tonne de valeur pondérée = tonne métrique $\times$ PAO)

- le potentiel d'appauvrissement global de l'ozone (PAGO = somme des valeurs pondérées de chacune des SAO).

\section{Résultats et discussion}

\section{Résultats}

Le Togo est essentiellement caractérisé par la consommation de :

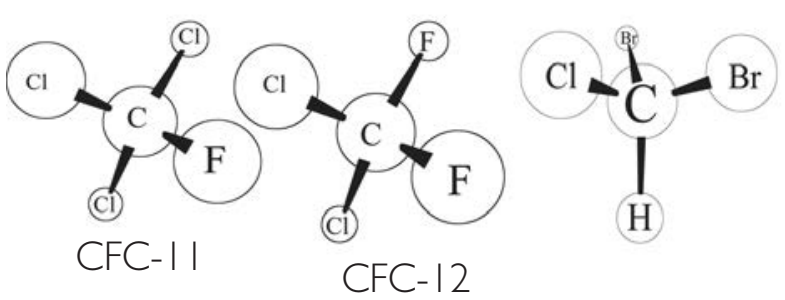


Sur la base des données générées du secteur de réfrigération, on obtient le résultat de la consommation en tonnes de valeurs pondérées des SAO essentielles importées au Togo en 2005 en appliquant la relation : tonne de valeur pondérée $=$ tonne métrique $\times$ PAO. D'où le tableau récapitulatif des résultats.

Tableau 14 : Consommation des SAO importés au Togo en 2005.

\begin{tabular}{|l|l|l|l|l|}
\hline SAO & 8,5 & PAO & $\begin{array}{l}\text { Consommation en tonnes } \\
\text { de valeur pondérée PAO }\end{array}$ & $\begin{array}{l}\text { Consommation par habitant en kg de } \\
\text { valeur PAO }\end{array}$ \\
\hline CFC-11 & 0,096 & 1,0 & 0,096 & $0,018.10^{3}$ \\
\hline CFC-12 & 916,704 & 1,0 & 916,704 & 0,176 \\
\hline CFC-22 & 326,437 & 0,055 & 17,954 & 0,0034 \\
\hline TOTAL & 1243,237 & & 934,754 & 0,179418 \\
\hline
\end{tabular}

Le résultat de la consommation par habitant entre 2000 et 2005 est obtenu à partir des données des douanes et consigné dans le tableau 15.

Tableau 15 : Consommation des SAO par habitant entre 2000 et 2005

\begin{tabular}{|l|l|l|l|l|l}
\hline & R-12 (tonnes de PAO) & R-22 (tonnes de PAO) & $\begin{array}{l}\text { Total SAO } \\
\text { (tonnes de PAO) }\end{array}$ & Population (habitants) & $\begin{array}{l}\text { Consommation par } \\
\text { habitant (tonne de } \\
\text { PAO) }\end{array}$ \\
\hline 2000 & 297,779 & 20,543 & 318,322 & 4635000 & $0,0689.10^{-3}$ \\
\hline 2001 & 263,223 & 18,159 & 281,382 & 4740000 & $0,0594.10^{3}$ \\
\hline 2002 & 140,933 & 9,723 & 150,656 & 4854000 & $0,0310.10^{-3}$ \\
\hline 2003 & 125,141 & 8,633 & 133,774 & 4970000 & $0,0269.10^{3}$ \\
\hline 2004 & 147,266 & 10,160 & 157,426 & 5090000 & $0,0309.1^{3}$ \\
\hline 2005 & 147,282 & 10,161 & 157,443 & 5212000 & $0,0302.10^{-3}$ \\
\hline
\end{tabular}

\section{Discussion}

\section{Cas du CFC-II}

Nos résultats ont donné le niveau de consommation de CFC-I I égal à 0,096 tonne métrique en 2005 alors que le Programme du Pays l'avait estimé à 0,49 tonne métrique en 1995. Ce qui donne la figure d'évolution suivante :

\section{Figure 3 : Evolution de consommation de CFC-11}

\section{Etude comparée de consommation de CFC-} 11: cas 2005

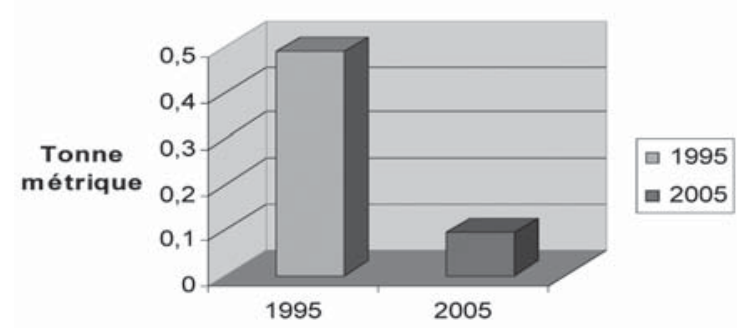

La consommation de CFC-I I en 2005, bien que faible par rapport à celle de 1995, n'est pas nulle conformément à l'ajustement au Protocole dans son article 2A alinéa 4 qui exige que pendant la période de douze mois commençant le ler janvier 1996, et ensuite pendant chaque période de douze mois, chacune des Parties veille à ce que son niveau calculé de consommation de CFC-I | soit réduit à zéro. La présence de CFC-II aujourd'hui au Togo s'explique par le commerce illicite.

\section{Cas du CFC-12}

La consommation totale de CFC-12 en 2005 s'élève à 916,70 tonnes selon nos résultats alors que le programme du Togo a estimé à 49,4l tonnes la consommation de 1995 et prévoit une réduction de moitié jusqu'en 2005 soit donc une consommation prévue à 24,705 tonnes pour l'année 2005.

La figure suivante présente ces comparaisons.

\section{Figure 4 : Etude comparée de consommation de CFC-12}

\section{Etude comparée de consom mation de CFC-12} en tonne métrique: cas 2005

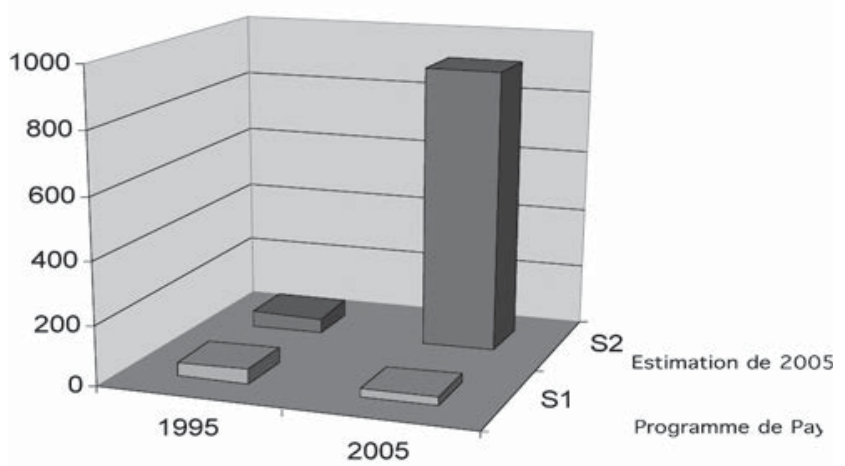

On déduit donc que nous sommes dans un rapport de 9 |6,70 / 24,705 soit 37, | I. Ce rapport très supérieur à l'unité indique bien que le Togo continue d'importer les matériels de consommation des SAO et en particulier du CFC-12

\section{Cas du CFC-22}

Figure 5 : Etude comparée de consommation de CFC-22

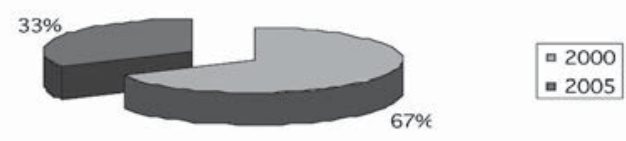

Le gel du CFC-22 est prévu pour l'an 2016 et l'élimination totale en 2040. Pour pouvoir faciliter son élimination dans l'avenir, l'ajustement au Protocole dans son article 2F alinéa 2 voudrait qu'en 2005, chacune des Parties veille à ce que son niveau calculé de consommation de CFC-22 n'excède pas $65 \%$ de celle de 1986.

Nos propres estimations indiquent une consommation en 2005 égale à33 \% de celle de 2000 . Les rapports $33 \%$ et $67 \%$ respectivement pour 2005 et 2000 permettent de dire que l'évolution de la consommation en CFC-22 est en conformité à l'ajustement. 


\section{Conclusion et recommandation}

En contribution à cette lutte pour la réduction allant à l'élimination progressive des SAO, notre étude nous a permis de constater au titre de l'année 2005 que le Togo a consommé : 0,096 tonne métrique de CFC-1।, 916,704 tonnes métriques de CFC-12 et 326,437 tonnes métriques de HCFC-22.

Pour réduire au mieux la consommation en SAO nous faisons les recommandations suivantes:

— élaborer le système de surveillance et d'octroi de licences

- réviser le système de codification douanière $\mathrm{SH}$

- renforcer les capacités douanières

— rendre les données accessibles à tous les niveaux

- créer une équipe de collecte variée et mobile

- éviter les élaborations unilatérales des actes

- procéder aux sensibilisations et formations de tous les acteurs.

\section{Bibliographie}

[1] La Convention de Vienne pour la protection de la couche d'ozone, Secrétariat de l'ozone, PNUE. Publication de Novembre 2001.

[2] Protocole de Montréal à des substances qui appauvrissent la couche d'ozone tel qu'ajusté et/ou amendé à Londres (1990), Copenhague (1992), Vienne (1995), Montréal (1997), Beijing (1999), Secrétariat de l'ozone, PNUE. Publication de 2000.

[3] Manuel sur la communication des données au titre du protocole de Montréal. PNUETIE, Publication des Nations Unies ISBN 92-807-1735-9 UNEP 1999.

[4] Programme de pays pour la protection de la couche d'ozone (Protocole de Montréal), Ministère du Développement rural, de l'Environnement et du Tourisme (République Togolaise). Version approuvée ; Juillet 1995.

[5] Rapport pour la deuxième réunion suivi de I'ODOSNET/AF-F à Brazzaville (Congo) du 18 au 19 Avril 1996. BNO du Togo.

[6] Amendement au Programme de pays pour la protection de la couche d'ozone (Mise en œuvre du Protocole de Montréal au Togo), (UNEP/CAP/IE), Ministère du Développement rural, de l'Environnement et du Tourisme (République togolaise). Août 1996.

[7] Code de l'environnement. Loi n 88-14 du 03 Novembre 1988.

[8] Protéger la couche d'ozone (PNUE). Vol. I. Frigorigènes ; 1992, 40 p.
[9] Protéger la couche d'ozone (PNUE), Vol. 2. Solvants revêtements et adhésifs ; 1992, 40 p.

[10] Protéger la couche d'ozone (PNUE).Vol. 3. Mousses ; 1992, 32 p.

[II] Protéger la couche d'ozone (PNUE), Vol. 4. Substances de lutte contre l'incendie ; 1992, 32 p.

[12] Manuel des instruments internationaux pour la protection de la couche d'ozone. Cinquième édition (2000).

[13] Le rôle de l'industrie hôtelière et touristique dans la protection de la couche d'ozone, Publication des Nations Unies. ISBN: 92-807-2066-X, Copyright 1998 PNUE.

[14] Elimination des SAO dans les pays en développement, Guide d'application des codes de bonnes pratiques, Secteur du froid. 1998.

[15] Système d'octroi des licences d'importation et d'exportation des SAO, Module des ressources. PNUE. 1998.

[1 6] Le Bulletin du programme ActionOzone du PNUE IE, Supplément spécial No. 3, Novembre 1995

[17] Supplément Spécial ActionOzone. Numéro 4. Novembre.

[18] Protection de la couche d'ozone : Elimination progressive des SAO dans les pays en développement, Manuel de formation des agents des douanes. PNUE. 2001.

[19] PNUE. Secrétariat de l'Ozone. http//ozone.unep.org/ french/Treaties_and_Ratification/2c_Ratification, consultation du 04/03/07. 